\title{
PLATAFORMA COLABORATIVA, ELÁSTICA, DE BAJO COSTO Y CONSUMO BASADA EN RECURSOS DE LA NUBE, CONTENEDORES Y MÓVILES PARA HPC
}

\author{
David Petrocelli ${ }^{12}$, Armando De Giusti ${ }^{34}$ y Marcelo Naiouf ${ }^{3}$ \\ ${ }^{1}$ Estudiante de Doctorado en Cs. de la Computación, Universidad Nacional de La Plata, 50 y 120, La Plata, Argentina \\ ${ }^{2}$ Profesor e Investigador de la Universidad Nacional de Luján, rutas 5 y 7, Luján, Argentina \\ ${ }^{3}$ Instituto de Investigación en Informática LIDI (III-LIDI), Facultad de Informática, Universidad Nacional de La Plata - \\ CIC-PBA 50 y 120 - La Plata-Buenos Aires - Argentina \\ ${ }^{4}$ CONICET - Consejo Nacional de Investigaciones Científicas y Técnicas
}

\begin{abstract}
RESUMEN
A la hora de resolver tareas de cómputo intensivo de manera distribuida y paralela, habitualmente se utilizan recursos de hardware (CPU/GPU) e infraestructura (Grid, Cluster, Nube) especializada para lograr un alto rendimiento. Sin embargo, estas arquitecturas consumen, inclusive en períodos ociosos, altos niveles de energía y requieren de una instalación y administración acorde provocando considerables gastos de inversión, mantenimiento y operación. En contraposición, gracias a la evolución constante y consumo masivo de los dispositivos móviles basados en procesadores ARM se extendió un paradigma alternativo. Estos equipos incrementan su capacidad, eficiencia, estabilidad y potencia a diario, mientras ganan masividad y mercado, conservando un costo, tamaño y consumo energético reducido. A su vez, cuentan con lapsos de ociosidad durante los períodos de carga, lo que representa un gran potencial que puede ser reutilizado. Con objeto de explotar esa capacidad y proponer un modelo alternativo para resolver trabajos HPC, se desarrolló y evaluó una plataforma distribuida, colaborativa, elástica y de bajo costo basada en contenedores eficientemente orquestados con Kubernetes, la cual recicla la capacidad ociosa de recursos móviles ARM y x86 tradicional. Para validar la escalabilidad, flexibilidad y rendimiento del sistema, se ejecutaron diversos escenarios concurrentes de transcoding de video. Los resultados muestran que la arquitectura desarrollada es una alternativa escalable, económica y elástica respecto a los modelos tradicionales.
\end{abstract}

\section{PALABRAS CLAVE}

Kubernetes, Contenedores, Microservicios, Computación en la Nube, Computación Móvil, Computación Colaborativa

\section{INTRODUCCIÓN}

Las compañías proveedoras de servicios de computación como Amazon, Microsoft o Netflix tienen grandes centros de datos para proporcionar la infinidad de servicios que ofrecen. Debido al continuo crecimiento en el tamaño, complejidad, y consumo de dichos centros, optimizar el uso, refrigeración y gasto energético, así como analizar arquitecturas alternativas (Mukta y Ahmed, 2020; Shuja et al., 2019) se convirtió en una preocupación principal a causa de su impacto directo en los costos y el medio ambiente (Masanet, 2020; Ahmed et al., 2019; Zaman et al., 2019). Como resultado, siguiendo el principio de que las necesidades de cómputo complejas a menudo se pueden dividir en problemas de menor tamaño (Czaja, 2018), la mayoría de las organizaciones han propuesto diferentes arquitecturas para implementar las características de escalabilidad, flexibilidad y concurrencia (Choudhury y Sridharan, 2020; Burns et al., 2019; Shkuro, 2019; Niall et al., 2016). En particular, el paradigma de computación voluntaria permite que los participantes compartan sus recursos informáticos en desuso y contribuyan a ejecutar proyectos computacionalmente costosos (Mengistu y Dunren, 2019). Sus pilares fundamentales consisten en asignar y administrar las tareas informáticas, e integrar un gran número de personas que voluntariamente donen su potencia de cálculo (Lavoie y Hendren, 2019). En la actualidad, muchas plataformas colaborativas constan de millones de usuarios activos. Por ejemplo, el proyecto Folding@Home de BOINC experimentó este semestre un increíble aumento en su capacidad de cómputo colaborativo debido a la participación masiva de investigadores, 
entidades y empresas para aportar sus ciclos de procesamiento en pos de avanzar con los estudios relacionados con el virus COVID-19 (Kratzke, 2020).

Por otro lado, a diferencia de la arquitectura x86, los procesadores ARM fueron concebidos teniendo en cuenta como pilar fundamental la eficiencia energética (Cristea et al., 2019; Pramanik et al., 2019), relegando en parte capacidad de cálculo (Ginny et al., 2020; Bhat et al., 2019) ya que estaban orientados a ser utilizados en móviles y microdispositivos alimentados mayormente por baterías. Estos equipos disponen de grandes lapsos de inactividad durante sus periodos de recarga, por lo que, si su capacidad se maneja adecuadamente, pueden ser reutilizados para convertirse en centros de datos masivos, consumiendo solo una fracción de la energía y otorgar una potencia informática similar a la de sus contrapartes (Gedawy et al., 2020; Barua y Mondal, 2019; Lavoie y Hendren, 2019; Hirsch et al., 2018; Schaffner et al., 2018; Mach y Becvar, 2017)

En este trabajo, se tomó el sistema desarrollado (Petrocelli et al., 2019; 2017) y se migró a una arquitectura de microservicios, lo que permitió que sus funciones fueran fragmentadas en servicios más pequeños y con responsabilidades mejor definidas (Hassan et al., 2020). A su vez, se integró una arquitectura de contenedores (Watada et al., 2020; Vayghan et al., 2019), específicamente Docker (Rad et al., 2017). Finalmente, con objeto de crear clusters de contenedores que se puedan administrar, desplegar, automatizar y escalar de manera integral, se incluyó el orquestador Kubernetes (K8s), tanto a nivel local (Dobies y Wood, 2020; Jha et al., 2019) como en la Nube (Arundel y Domingus, 2019), lo que garantiza un uso eficiente de los recursos, costos y energía (Zhong y Buyya, 2020; Dewi et al., 2019; Townend et al., 2019).

Las pruebas sobre el sistema, a diferencia de nuestros artículos anteriores (Petrocelli et al., 2019; 2017) donde se analizó el consumo energético y performance individual de los dispositivos, se diseñaron con objeto de verificar la capacidad de la plataforma para escalar ante diversos escenarios de carga y saturación. Al igual que en los estudios anteriores, la tarea HPC implementada fue la transcodificación de vídeo bajo demanda, de manera distribuida y paralela. El análisis de los resultados permite considerar a este sistema como una alternativa, escalable y de bajo costo para resolver tareas de cómputo intensivo.

El trabajo está organizado de la siguiente manera. En la Sección 2, se detalla el protocolo, tecnologías y funciones desarrolladas. En el Apartado 3, se explica la metodología, métricas, escenarios y pruebas a realizar. En la Sección 4, se presenta un análisis pormenorizado de los resultados. En el Apartado 5 se describen las conclusiones y, finalmente, en la Sección 6 se describen los trabajos futuros.

\section{PROTOCOLO, TECNOLOGÍAS Y FUNCIONES DESARROLLADAS}

La arquitectura desarrollada está compuesta por: a) Microservicios Dockerizados para la administración y gestión de la plataforma; b) Servicios de colas (tareas) y base de datos (estadísticas) Dockerizadas; c) Orquestación, escalado, replicación y automatización de contenedores a través de Kubernetes y d) Trabajadores móviles Android ARM y x86 Dockerizados (Figura 1), que se detallan a continuación.

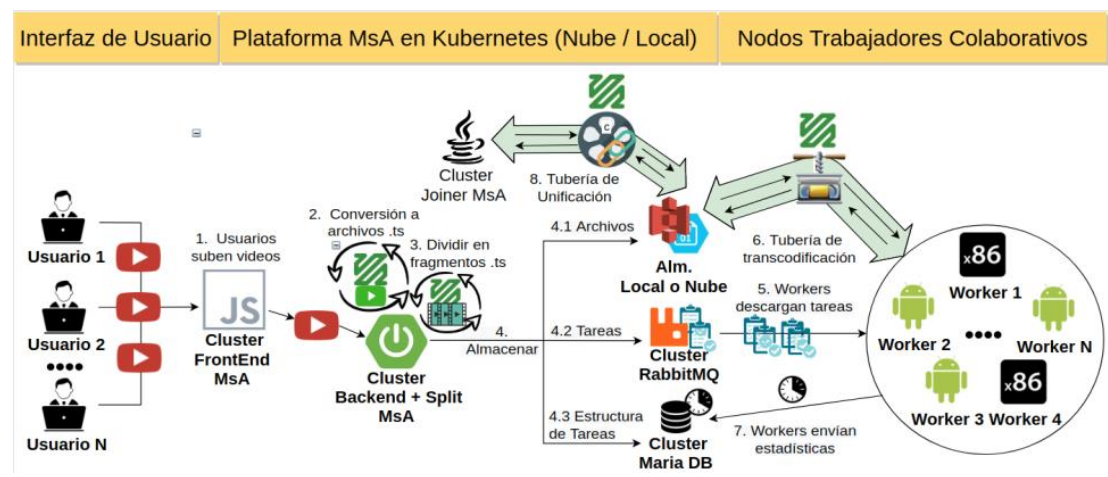

Figura 1. Arquitectura y componentes que forman parte de la arquitectura desarrollada

a) Microservicios Dockerizados para la administración y gestión de la plataforma

Para el desarrollo y despliegue del sistema se adoptaron herramientas, tecnologías y arquitecturas que permitieran aprovechar al máximo los recursos informáticos disponibles (locales, Nube y móviles) para realizar las tareas HPC. Se utilizó una arquitectura basada en microservicios, lo que permitió obtener una 
mayor flexibilidad a la hora de incluir nuevos cambios, actualizaciones y/o dependencias, adoptando un esquema que puede rápidamente ser replicado y escalado (Rudrabhatla, 2020; Baskarada et al., 2018). A su vez, estas funciones se dockerizaron, lo que permite incluir todos los recursos necesarios para que los mismos sean portables, ligeros y puedan ejecutarse de manera encapsulada dentro de una sola imagen completamente independiente del servidor anfitrión (Rad et al., 2017). Para ello, a través del framework Spring Boot se generaron APIs REST, de modo que los clientes, servicios y nodos puedan interactuar a través de mensajes JSON vía HTTP. A su vez, estos microservicios se conectan, registran, auditan y actualizan los trabajos en el sistema de colas (RabbitMQ) y los datos estadísticos (tiempos de ejecución) en la base de datos (MariaDB). Las funciones del Backend de administración y gestión del sistema se dividen en:

a.1) Web + Split: Con esta funcionalidad se convierten las solicitudes de los clientes en tareas de procesamiento distribuido (archivos de video fuente y parámetros de transcodificación). Una vez recibidos, los vídeos se convierten al formato Transport Stream (.ts). Esta conversión permite, posteriormente, fragmentar el video en partes más pequeñas e independientes entre sí, lo que posibilita una recodificación, transmisión y reproducción de vídeo distribuida (Rakesh et al., 2020; Sani et al., 2017). Una vez divididos, los segmentos se guardan en un almacenamiento de bloques en la Nube de bajo costo, cómo Azure Blob Storage o Amazon S3 (Zouheir y Hassan, 2018), o en un sistema de archivos local, dependiendo el ambiente de ejecución y se generan los procesos de compresión utilizando los parámetros que definió el usuario. Siendo N perfiles de compresión y M partes, se generan entonces NxM trabajos los cuales guardan en el sistema de colas persistente. Su uso permite que las actividades se procesen de manera asincrónica por parte de los nodos trabajadores. También se registra en la base de datos la estructura de la tarea para almacenar las métricas estadísticas (cantidad de partes, partes completas y tiempos de ejecución). Las actividades de conversión y particionado se realizan utilizando el binario de FFMpeg y las operaciones sobre los archivos con la herramienta Curl.

a.2) Joiner + Unifier: Los servicios Joiner consultan continuamente la base de datos para validar cuando un trabajo de compresión ha finalizado. Si existe un caso, genera un registro en el sistema de colas donde un microservicio de tipo Unifier la tomará y aplicará un proceso de unificación a través de la librería FFMpeg. Una vez completado, se guarda el archivo final y se registra la información estadística.

a.3) FileServer: Es un servidor de archivos NFS vía API REST utilizado en caso de desplegar la arquitectura en un ambiente de Kubernetes local y no disponer un almacenamiento externo pago como Amazon S3 o Azure Blob Storage, el cual recibe las peticiones curl y persiste los archivos de forma segura y redundante.

b) Servicios de colas (tareas) y base de datos (estadísticas) Dockerizadas

Se utilizan las imágenes de RabbitMQ y MariaDB Galera de Bitnami, para crear un sistema de colas y base de datos auto escalable y redundante. RabbitMQ se utiliza para registrar, publicar y distribuir los trabajos (jobs) de forma segura y asincrónica a los nodos de procesamiento. La alta disponibilidad está garantizada por las políticas HA de RabbitMQ y su manejo en Kubernetes. También se implementa un modelo de confirmación manual de proceso completado (ACK) donde, en caso de que ocurra un error del cliente o servidor, o expire una marca de tiempo, RabbitMQ devuelve el trabajo a la cola sin perder o corromper datos. El motor MariaDB se usa para registrar información de estado y estadística de las tareas (parámetros, fragmentos, punto de acceso al recurso, nodos de procesamiento, tiempos de ejecución). La alta disponibilidad se garantiza a través de la topología multimaestro (activo-activo) de Galera en Kubernetes.

c) Orquestación, escalado, replicación y automatización de contenedores a través de Kubernetes

Para correr, alojar, orquestar, controlar y monitorear la arquitectura Dockerizada, se utiliza el orquestador Kubernetes (Burns et al, 2019). En la Nube, a través de infraestructuras SaaS de AWS (Ifrah, 2019) y Azure (Buchanan et al, 2019) y localmente con clústeres Kubernetes (K8s) construidos con Kubeadm. Independientemente del escenario, los sistemas de colas y base de datos se aíslan en un segmento de recursos reservados (namespaces) para garantizar su funcionamiento como pilar del sistema. Al resto de los microservicios (deployment - pods) se le definen cuotas, reservas y mecanismos de escalabilidad automática (Thanh-Tung et al, 2020) basados en una cantidad mínima de contenedores (pods $=3$ ) y umbrales de utilización de memoria y CPU (75\%), lo que garantiza estabilidad, redundancia, flexibilidad y una distribución de carga eficiente. En el caso particular de la Nube, se habilita el autoescalado de hosts K8s para aprovechar la elasticidad de esta infraestructura y optimizar los costos y consumo energético asociados. d) Trabajadores móviles Android ARM y x86 Dockerizados

Los nodos trabajadores se desarrollaron utilizando el lenguaje Java. Para la versión móvil con el SDK nativo (compatibilidad Android 7+, API Level 24) y Dockerizando para la versión x86. Con esta aplicación 
los nodos descargan, sincronizadamente, tareas del sistema de colas para ser transcodificadas. Una vez obtenida, con la url del fragmento de video y los perfiles de compresión se inicia la actividad de transcodificación utilizando FFmpeg y tuberías (pipelines) concurrentes. Mientras se está descargando el video con una petición curl GET, FFmpeg toma el flujo de datos y va transcodificando mientras escribe el flujo de salida en el almacenamiento destino con una petición curl PUT. Como resultado, las operaciones de E/S sobre disco y el uso de memoria se reducen drásticamente, mejorando el rendimiento del sistema. Finalizado este proceso, se confirma la tarea realizada en el sistema de colas y se actualiza la información estadística (tiempos) en la base de datos.

\section{DEFINICIÓN DE ESCENARIOS DE PRUEBA}

La plataforma se desplegó en las Nubes públicas de Azure (AKS) y Amazon (EKS) con Kubernetes versión 1.17.9 para validar el funcionamiento de los nodos trabajadores móviles a través de Internet, registrando los recursos de hardware y versión de Android de los dispositivos. Al mismo tiempo, se ejecutaron pruebas de carga y estabilidad bajo un entorno controlado y homogéneo de Kubernetes y Docker local, con objeto de evitar costos durante el proceso de prueba. Este ambiente se construyó con 9 equipos Blade HP ProLiant BL460c G8. Cada uno incluye 2 procesadores Intel Xeon E5-2640 v2 (2.0 Ghz x 16 cpus), alojando una máquina virtual de 32 cores y $32 \mathrm{gb}$ de ram con SO Debian 10.4, Docker 19.03.12 y Kubernetes versión 1.17.9 y con una placa de red de 1 Gbps. Los videos utilizados para ejecutar las pruebas se dividieron en fragmentos de 10 segundos (Rakesh et al., 2020) y se transcodificaron utilizando el códec H.264/AVC (Shi y Sun, 2019) a través de cinco perfiles de compresión (Tabla 1). Esto permite generar videos en diferentes calidades, adecuadas para distintos dispositivos y ancho de banda (Raake et al., 2017; Sani et al., 2017).

Tabla 1. Perfiles utilizados para la trascodificación de videos con H.264/AVC

\begin{tabular}{|l|l|l|l|l|l|l|}
\hline Perfiles de transcodificación & Tamaño de Pantalla & Codec de Video & Bitrate de Video & Perfil de Video & Nivel del Códec & Opciones del Codec \\
\hline Perfil 2K & $2560 \times 1440$ & AVC $\times 264$ & $7800 \mathrm{Kbps}$ & High & L@5.1 & slower \\
\hline Perfil Full-HD 1080p & $1920 \times 1080$ & AVC $\times 264$ & $3900 \mathrm{Kbps}$ & High & L@4.1 & slow \\
\hline Perfil HD 720p & $1280 \times 720$ & AVC $\times 264$ & $2000 \mathrm{Kbps}$ & Main & L@4.1 & medium \\
\hline Perfil 480p & $852 \times 480$ & AVC $\times 264$ & $900 \mathrm{Kbps}$ & Main & L@3.1 & fast \\
\hline Perfil 240p & $424 \times 240$ & AVC $\times 264$ & $500 \mathrm{Kbps}$ & Baseline & L@3.0 & ultrafast \\
\hline
\end{tabular}

Se diseñaron y ejecutaron tres escenarios de carga. Los dos primeros validaron la capacidad de la plataforma para escalar al realizar múltiples tareas de transcodificación. Para ello, se definieron diversos ambientes de prueba, donde se varió el material y los nodos trabajadores, registrando los tiempos de procesamiento. En el primero (E.C.1), se utilizó una carga fija y se varió la cantidad y tamaño de los nodos trabajadores. En el segundo (E.C.2), se experimentó con material variable y se fijó la cantidad y tamaño de los nodos de procesamiento. El tercero (E.C.3), se diseñó y ejecutó con el objeto de verificar la flexibilidad del sistema ante solicitudes de transcodificación concurrente, registrando el tiempo de recepción y preparación de las tareas.

Para E.C.1 y E.C.2, se definieron tres tipos de nodos trabajadores, limitando sus recursos a través de la API de Docker: low-cpu con 2 cpus y $1.5 \mathrm{gb}$ de memoria, mid-cpu con 4 cpus y $3 \mathrm{gb}$ y high-cpu con 8 cpus y $6 \mathrm{gb}$, siguiendo las definiciones más tradicionales de los dispositivos móviles, corriendo sobre cinco configuraciones de clusters: 2, 4, 8, 16 y 32 nodos homogéneos. En las pruebas E.C.1 y E.C.2 se utilizaron videos MP4 codificados en perfil High @L5.2 AVC x264, con un tamaño de pantalla de 3840x2160 (4k), con 24, 30 o 60 cuadros por segundo, obtenidos de Vimeo y Demolandia.

En la prueba E.C.1 se definió una carga de trabajo (WK) de 5 videos fuente de 1 a 2 minutos (WK 1 en Tabla 2) con una alta tasa de bits, donde se aplicaron los perfiles de compresión para cada video (Tabla 1), generando así 25 tareas de compresión. Los trabajadores variaron entre 2, 4, 8, 16 y 32 de cada tipo de nodo.

En el escenario E.C.2 se fijaron 32 nodos trabajadores del tipo high-cpu. Se ejecutaron diferentes sets de videos de una duración de 1 a 30 minutos (WK 2 a WK 6 en Tabla 2) según la siguiente fórmula: $\mathrm{TC}=25 \mathrm{x}$ M. Dónde, 25 surge de los 5 videos fuente de cada WK (Tabla 2) multiplicado por los 5 perfiles de compresión (Tabla 1); y M es el multiplicador de tareas. En las pruebas ejecutadas, M varió entre 10 y 60 , resultando entre 250 y 1500 tareas por cada carga de trabajo. 
Tabla 2. Videos seleccionados para las cargas de trabajo (Workloads) utilizados para los escenarios de prueba

\begin{tabular}{|c|c|c|c|c|c|c|c|c|c|c|c|c|c|c|c|c|c|}
\hline \multirow{2}{*}{$\begin{array}{l}\text { WK } 1 \\
\text { Video }\end{array}$} & \multicolumn{2}{|c|}{ High-Bitrate } & \multirow{2}{*}{\begin{tabular}{|l|} 
WK 2 \\
Video \\
\end{tabular}} & \multicolumn{2}{|c|}{$1 \mathrm{~min} 4 \mathrm{k}$} & \multirow{2}{*}{\begin{tabular}{|l|} 
WK 3 \\
Video \\
\end{tabular}} & \multicolumn{2}{|c|}{$2 \min 4 k$} & \multirow{2}{*}{\begin{tabular}{|l|} 
WK 4 \\
Video \\
\end{tabular}} & \multicolumn{2}{|c|}{$4 \min 4 k$} & \multirow{2}{*}{\begin{tabular}{|l|} 
WK 5 \\
Video \\
\end{tabular}} & \multicolumn{2}{|c|}{$10 \min 4 \mathrm{k}$} & \multirow{2}{*}{\begin{tabular}{|l|} 
WK 5 \\
Video \\
\end{tabular}} & \multicolumn{2}{|c|}{$30 \mathrm{~min} 4 \mathrm{k}$} \\
\hline & Mbps & GB & & Mbps & GB & & Mbps & GB & & Mbps & GB & & Mbps & GB & & Mbps & GB \\
\hline Norway & 33 & 0.27 & Chicago & 18.6 & 0.13 & Ghostflight & 18.6 & 0.18 & Memories & 14.3 & 0.42 & Laule & 10.8 & 0.8 & Atmos & 15.8 & 3.37 \\
\hline Switzerland & 19.9 & 0.32 & Urederra & 19.4 & 0.14 & Crrousel & 16.4 & 0.24 & Humanity & 15.6 & 0.45 & Killing & 11.2 & 0.83 & Bosques & 19.2 & 4.10 \\
\hline PanamaX & 22.1 & 0.41 & \begin{tabular}{|l|} 
Portugal \\
\end{tabular} & 19.9 & 0.15 & Brasil & 19.5 & 0.32 & Alpine & 18.3 & 0.54 & Theresa & 14.1 & 1 & Sean & 22 & 4.68 \\
\hline Landscape & 125 & 1.68 & Wonder & 22 & 0.18 & Pilot & 22.2 & 0.33 & Lake & 19.9 & 0.56 & German & 14.6 & 1,04 & longT & 22.7 & 4.82 \\
\hline Europe & 125 & 02.08 & Alaska & 22 & 0.18 & Cambridge & 23.2 & 0.34 & World & 22.8 & 0.67 & Vida & 21.1 & 1.50 & Sydney & 23.6 & 5,05 \\
\hline
\end{tabular}

La prueba E.C.3 se llevó a cabo a través de un script bash que generó entre 250 y 1500 peticiones curl concurrentes, utilizando el video "Chicago" del set WK2 (Tabla 2). La cantidad de sesiones paralelas se eligió teniendo en cuenta la capacidad de la red del laboratorio de pruebas. Las ejecuciones se realizaron sobre diversas configuraciones de tamaño y cantidad de servidores web. Se definieron 3 tipos de nodos siguiendo las configuraciones más tradicionales de las instancias de Amazon EC2: a1.medium con 1 cpus y 2 gb de memoria ram; a1.large con 2 cpus y $4 \mathrm{gb}$; y a1.xlarge con 4 cpus y $8 \mathrm{gb}$, limitando sus recursos a través de la API de Docker, corriendo sobre configuraciones de 1, 2, 4 y 8 nodos web homogéneos.

\section{ANÁLISIS DE RESULTADOS}

Luego de desplegar la plataforma en la Nube a través de los servicios de Kubernetes de AWS y Azure, y publicar reiteradas veces las cargas definidas en WK1 (Tabla 2), se realizaron pruebas de compatibilidad del nodo trabajador móvil en distintos dispositivos (Tabla 3). Se corroboró que la aplicación funciona correctamente en equipos que tengan instalado Android 7 (lanzado en 2016) o superior (API Level 24) ya sea con arquitectura de 32 o 64 bits. También se comprobó que la inclusión de tuberías Linux en la aplicación evitó la sobrecarga de memoria, permitiendo ejecutar cada tarea sin errores, independientemente de la potencia del dispositivo. Al mismo tiempo, se probó el funcionamiento del sistema y sus servicios de equilibrio de carga, escalado horizontal, manejo de errores y alta disponibilidad.

Tabla 3. Dispositivos móviles utilizados para corroborar el funcionamiento de la aplicación de nodo trabajador

\begin{tabular}{|c|c|c|c|c|c|c|c|c|c|c|c|c|c|c|c|c|c|}
\hline Айо & Modelo & CPU & API & Arq. & \begin{tabular}{l|l} 
so & Ai \\
\end{tabular} & Año & Modelo & CPU & API & Arq. & so & Año & Modelo & CPU & & Arq. CPU & Ti \\
\hline 2020 & Xiaomi Note 10 Lite & Snapdragon $730 \mathrm{G}$ & 29 & aarch64 & & 2019 s & Samsung A 50 & Exynos 9610 & 28 & armv81 9 & 9 & 2017 & Samsung S8 & Exynos 8895 & 28 & aarch64 9 & \\
\hline 2020 & Samsung Galaxy 520 & Exynos990 & 29 & aarch64 & & $2019 x$ & Xiaomi Redmi Note 7 & Snapdragon 660 & 28 & aarch64 9 & 9 & 2017 & Samsung Galaxy A5 & Exynos 7880 & 26 & aarch64 8 & 8 \\
\hline 2019 & Samsung Galaxy $\mathrm{S}_{10+}$ & Exynos 9820 & 29 & $\operatorname{arch} 64$ & & \begin{tabular}{l|l}
018 & Se \\
\end{tabular} & msung A6+ & Snapdragon 450 & 29 & armv81 1 & 1022 & 2017 & Moto E4 Plus & Mediatek MT6737 & 25 & \begin{tabular}{|l|l} 
armv71 & 7 \\
\end{tabular} & 7.1 \\
\hline 2019 & OnePlus $7 \pi$ & Snapdragon $855+$ & 29 & aarch64 & & $2018 \mathrm{~S}$ & Samsung J8 & Snapdragon 450 & 29 & \begin{tabular}{|l|l|l|l|l} 
armv81 & 1
\end{tabular} & 102 & 2016 & Samsung Galaxy 57 & Exynos 8890 & 26 & aarch64 8 & 8.1 \\
\hline 2019 & Samsung Galaxy A20 & Exynos 7884 & 29 & aarch64 & 1020 & \begin{tabular}{l|l}
2018 & $\mathrm{~s}$ \\
\end{tabular} & Samsung Gal & \begin{tabular}{|l|} 
Exynos 9810 \\
\end{tabular} & 29 & aarch64 1 & 102 & 2016 & Samsung J7 Prime & $y n o s 7870$ & 27 & \begin{tabular}{|l|l} 
armv81 & 8 \\
\end{tabular} & 8.1 \\
\hline 2019 & Samsung Ge & Exynos 7904 & 29 & aarch64 & & 2018 M & Moto One & Snapdragon 625 & 29 & $\operatorname{arch} 641$ & 102 & 2016 & Samsung Gal J5 Prime & Exynos 7570 & 26 & \begin{tabular}{|l|l|} 
armv81 & 8 \\
\end{tabular} & 8.0 \\
\hline 2019 & Xiaomi R. Note 8 Pro & Mediatek G90T & 29 & armv81 & 1020 & $2018 \mathrm{~N}$ & Moto G6 Plus & Snapdragon 630 & 28 & $\operatorname{arch} 649$ & 9 & 2016 & Moto G4 Plus & Snapdragon 617 & 24 & \begin{tabular}{|l|l|} 
armvv71 7 \\
\end{tabular} & 7.0 \\
\hline 2019 & Moto G7 Play & Snapdragon 632 & 29 & armv71 & $\begin{array}{l}1020 \\
20\end{array}$ & \begin{tabular}{l|l}
0018 & $\mathrm{M}$ \\
\end{tabular} & Moto E5 & Snapdragon 425 & 26 & 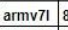 & 8 & 2015 & Samsung $\$ 5$ New Ed. & Exynos 7580 & 23 & armv71 6 & 6.0 \\
\hline 2019 & Samsung A70 & Snapdragon 675 & 29 & armv81 & $10 \mid 20$ & 2017 G & Google pixel $2 \mathrm{X}$ & Snapdragon 835 & 29 & $\operatorname{aarch} 64 \mid 1$ & 102 & 2015 & Moto G3 & Snapdragon 410 & 23 & armv71 & 6.0 \\
\hline 2019 & Mo & on 665 & 28 & aarch64: & $\begin{array}{l}9 \\
9\end{array}$ & 2017 M & Moto & Snapd & 29 & \begin{tabular}{l|l} 
armv71 & 1 \\
\end{tabular} & \begin{tabular}{|l|l}
102 & \\
\end{tabular} & 2014 & ind Prime & 450 & t & armv71 & 5.0 \\
\hline 2019 & Motorola E6 6 & ragon 435 & \begin{tabular}{|l|}
28 \\
\end{tabular} & armv71 & \begin{tabular}{l|l|l}
9 & 20 \\
\end{tabular} & \begin{tabular}{l|l}
2017 & $\mathrm{~s}$ \\
\end{tabular} & Samsung J7 pro & Exynos 7870 & 28 & armv81 & & 2013 & Sony Xperia Z1 & Snapdragon 800 & 22 & armv7ı & A \\
\hline
\end{tabular}

E.C.1: El rendimiento obtenido estuvo relacionado con las capacidades de cada categoría de nodos y con la complejidad del perfil aplicado. En todos los casos, los fragmentos de video se transcodificaron en menos de 2 minutos (Figure 2).
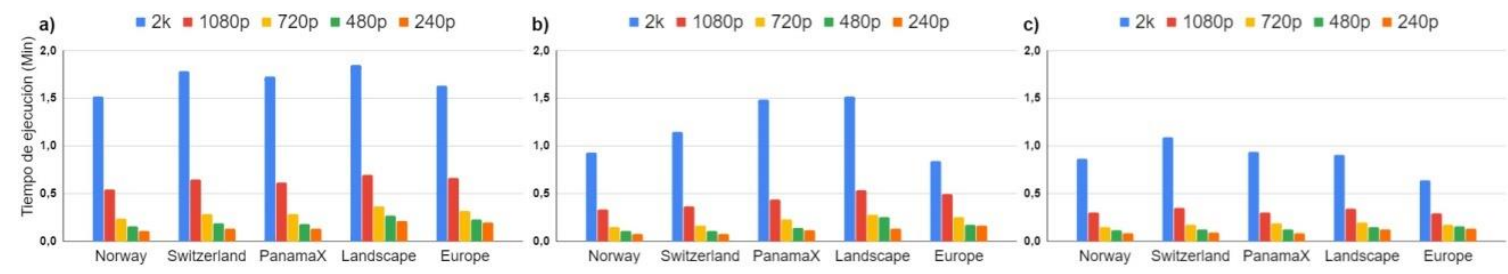

Figura 2. Tiempo de trascoding (minutos) para videos WK1 en nodos: a) low-cpu; b) mid-cpu; c) high-cpu

Los resultados indican, considerando el perfil más complejo (2k), que al aumentar la capacidad de los dispositivos trabajadores de low a mid-cpu, los tiempos de ejecución se reducen en un $31 \%$. Esto mismo se observó al incrementar de low-cpu a high-cpu, donde la reducción alcanza en promedio, un 47\% (Figura 3). Con respecto a los tiempos totales de ejecución sobre las diversas topologías de clusters, se puede visualizar que, al duplicar la cantidad de nodos el tiempo total de compresión se reduce en promedio, por cada salto, entre un $40 \%$ y $45 \%$. Si se compara la configuración inicial de 2 nodos con la de 32 colaboradores, el tiempo necesario se reduce de 8 a 10 veces (Figura 4). 


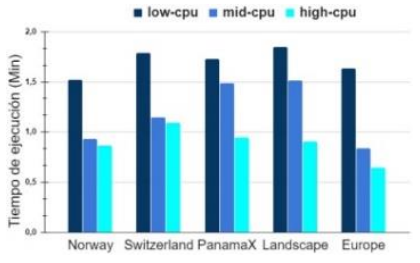

Figura 3. Tiempo de ejecución promedio por tipo de nodo

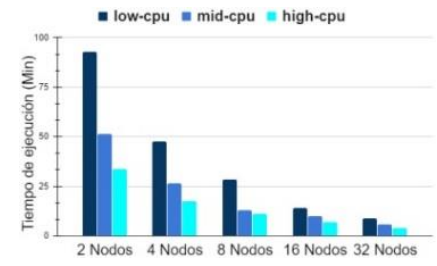

Figura 4. Tiempo total para diferentes cantidades de nodos

E.C.2: A través de los escenarios definidos, se verificó que la plataforma es flexible y escalable ante cargas de trabajo crecientes. Se probó que al mantener la cantidad de nodos de procesamiento fija y aumentar el número y la duración de los vídeos (más partes a procesar), se incrementa el tiempo total de procesamiento (Figura 5 a Figura 9). Con el objeto de caracterizar y poder extrapolar los resultados, se ajustó cada ejecución con una recta de tendencia de carácter lineal según $\mathrm{f}(\mathrm{x})=\mathrm{a}^{*} \mathrm{x}+\mathrm{c}$, donde a es la pendiente y c la constante. Como resultado, a medida que se complejizan los escenarios de prueba y por lo tanto se genera un acumulamiento de fragmentos a procesar con una capacidad fija, el valor de la pendiente (a) se vuelve mayor, impactando directamente en la performance y tiempos de respuesta (Figura 10).
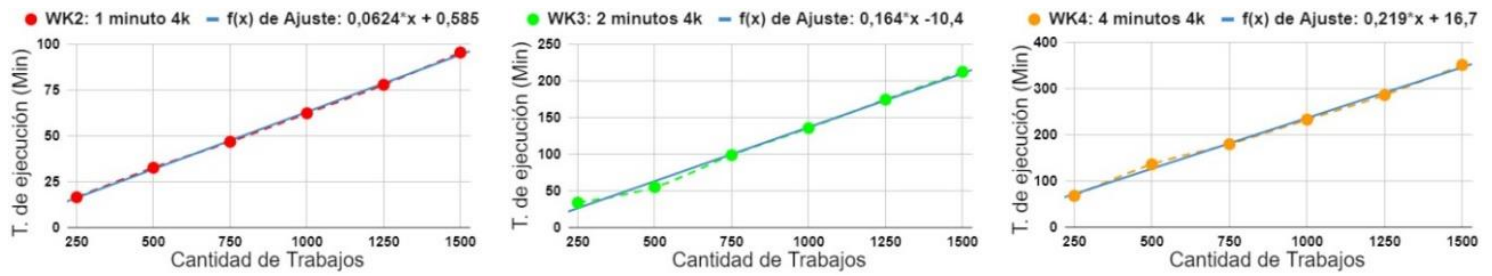

Figura 5. Tiempos de ejecución -WK2

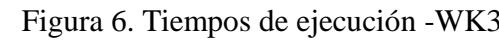

Figura 7. Tiempos de ejecución -WK4
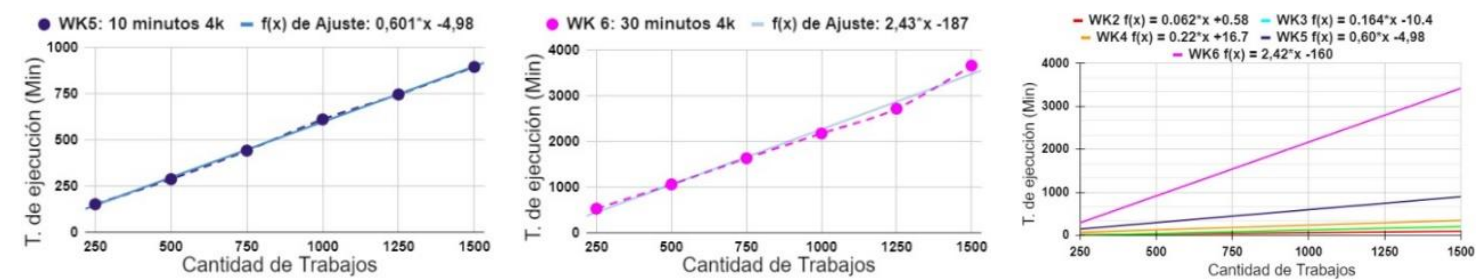

Figura 8. Tiempos de ejecución -WK5 Figura 9. Tiempos de ejecución -WK6 Figura 10. Rectas de Ajuste WK2-6

E.C.3: Se validó que el sistema responde correctamente a las peticiones concurrentes de transcodificación de video establecidas. El tiempo total de recepción y preparación de los trabajos estuvo relacionado con las capacidades de la red y las características y cantidades de nodos servidores (Fig. 11). Se probó que la complejidad de los procesos de lectura del buffer de comunicación, conversión del flujo a un archivo Transport Stream (.ts), su fragmentación a partes de 10 segundos y comunicación con sistema de cola, base de datos y sistema de archivos, todo de manera concurrente, conllevan a un uso intensivo de CPU, memoria y disco.

Si bien los nodos a1.medium atendieron a las solicitudes concurrentes sin errores, se consideran una arquitectura poco escalable debido a los tiempos de respuesta obtenidos y a sus pendientes de crecimiento pronunciadas, al menos para las configuraciones de nodos utilizadas. Los casos a1.large y a1.xlarge con arquitecturas de al menos 2 nodos son lo suficientemente robustas ante una demanda concurrente de tareas.
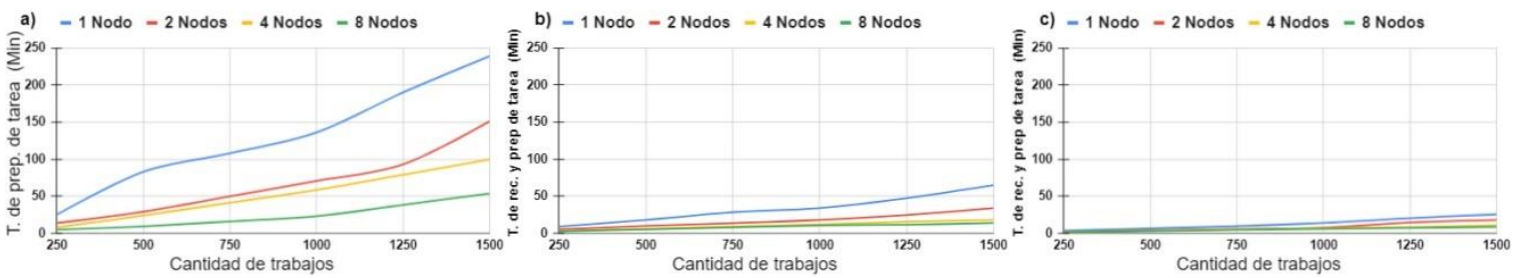

Figura 11. Escenario de carga E.C.3 para configuraciones de web server: a) a1.medium; b) a1.large; y c) a1.xlarge 


\section{CONCLUSIONES}

En este trabajo se desarrolló, desplegó y evaluó una plataforma colaborativa, distribuida, escalable y de bajo costo basada en microservicios, contenedores orquestados por Kubernetes y servicios en la Nube, la cual reutiliza la capacidad ociosa de los dispositivos móviles y x86 para realizar tareas informáticas HPC.

Se probó, a través de la experimentación sobre tareas de transcoding de video, que el sistema: a) responde de manera elástica y flexible ante diversas cargas de trabajo; b) resuelve las tareas de procesamiento de forma concurrente sin importar el volumen de carga y la cantidad y potencia de los nodos trabajadores.

Se evaluó también que: c) la aplicación de procesamiento móvil es compatible con cualquier dispositivo que tenga Android 7 o superior instalado; y d) si bien la performance está relacionada con la capacidad del equipo, la inclusión de tuberías Linux reduce la cantidad de memoria necesaria, permitiendo ejecutar cada tarea sin errores independientemente de su potencia.

En base a los resultados obtenidos, se concluye que la arquitectura desarrollada constituye una alternativa conveniente, escalable y de bajo costo para resolver tareas de cómputo intensivo.

\section{TRABAJOS FUTUROS}

Con objeto de extender las funcionalidades y el análisis de la plataforma, se pretende: a) incluir nuevos servicios de procesamiento paralelo como por ejemplo: procesamiento de señales, simulaciones físicas, recuperación de información, análisis masivo de datos, etc. que permitan realizar una mayor cantidad de pruebas y análisis del comportamiento del sistema; b) implementar un clasificador de tareas y nodos trabajadores que permita asignar las actividades y los equipos de procesamiento de manera inteligente; y c) implementar un modelo de incentivos basado en créditos el cual beneficie a los usuarios que colaboren con sus dispositivos durante los momentos de inactividad.

\section{REFERENCIAS}

Ahmed K. et al, 2019. Electrical Energy Consumption Model of Internal Components in Data Centers. 2019 IEEE PES Innovative Smart Grid Technologies Europe (ISGT-Europe), Bucharest, Romania, pp 1-5

Arundel J. y Domingus J., 2019. Cloud Native DevOps with Kubernetes. O'Reilly Media, California, USA

Barua H. y Mondal D., 2019. Approximate Computing: A Survey of Recent Trends - Bringing Greenness to Computing and Communication. The Journal of the Institution of Engineers: Electronics and Telecommunication, pp 619-626

Baskarada S., et al, 2018. Architecting Microservices: Practical Opportunities and Challenges. Journal of Computer Information Systems, Volume 60 - No 5, pp 428-436

Bhat G., et al, 2019. Power and Thermal Analysis of Commercial Mobile Platforms: Experiments and Case Studies. 2019 Design, Automation \& Test in Europe Conference \& Exhibition (DATE), Florence, Italy, pp 144-149

Buchanan S., et al, 2019. Introducing Azure Kubernetes Service: A Practical Guide to Container Orchestration. Apress, California, USA.

Burns B., et al, 2019. Kubernetes Up and Running: Dive into the Future of Infrastructure (2nd edition). O'Reilly Media, California, USA

Choudhury D. y Sridharan C., 2020. Scaling Microservices - Platform Engineering for Distributed Systems. O'Reilly Media, California, USA

Cristea V., et al, 2019. Mobile Phones and Energy Consumption. En Green IT Engineering: Social, Business and Industrial Applications (1. ${ }^{\mathrm{a}}$ ed., pp 243-271) Springer Nature, Cham, Switzerland

Czaja L., 2018. Introduction to Distributed Computer Systems: Principles and Features (1st edition). Springer Nature, London, UK.

Dewi L., et al, 2019. Server Scalability Using Kubernetes. 2019 4th Technology Innovation Management and Engineering Science International Conference (TIMES-iCON), Bangkok, Thailand, pp 1-4.

Dobies J. y Wood J, 2020. Kubernetes Operators - Automation the container Orchestration Platform. O'Reilly Media, California, USA

Gedawy H., et al, 2020. RAMOS: A Resource-Aware Multi-Objective System for Edge Computing. IEEE Transactions on Mobile Computing

Ginny, et al, 2020. Smartphone processor architecture, operations, and functions: current state-of-the-art and future outlook: energy performance trade-off. The Journal of Supercomputing (J SUPERCOMPUT). 
Hassan S., et al, 2020. Microservice transition and its granularity problem: A systematic mapping study. Software Practice and Experience, Volume 1 - No 1, pp 1-36.

Hirsch M., et al, 2018. Augmenting computing capabilities at the edge by jointly exploiting mobile devices: A survey. Future Generation Computer Systems (FUTURE GENER COMP SY), pp 644-662.

Ifrah S., 2019. Deploy Containers on AWS: With EC2, ECS, and EKS. Apress. California. USA.

Jha D., et al, 2019. A study on the evaluation of HPC microservices in containerized environment. Concurrency and Computation: Practice and Experience, pp 1-18.

Kratzke N., 2020. Volunteer Down: How COVID-19 Created the Largest Idling Supercomputer on Earth. Future Internet, Basel Volume 12, No. 6.

Lavoie E. y Hendren L., 2019. Personal Volunteer Computing. CF '19: Proceedings of the 16th ACM International Conference on Computing Frontiers, pp 240-246.

Mach P. y Becvar Z., 2017. Mobile Edge Computing: A Survey on Architecture and Computation Offloading. IEEE Communications Surveys \& Tutorials, Volume: 19 - No 3, pp 1628-1656

Masanet E., 2020. Recalibrating global data center energy-use estimates. Science, Volume 367, Issue 6481, pp 984-986

Mengistu T. y Dunren C., 2019. Survey and Taxonomy of Volunteer Computing. ACM Computing Surveys 52, pp 1-35.

Mukta T. y Ahmed I., 2020. Review on E-Waste Management Strategies for Implementing Green Computing. International Journal of Computer Applications, Volume 177 - No. 44, pp 45-52.

Niall R., et al, 2016. Site Reliability Engineering - How Google Runs Production Systems. O'Reilly Media, California, USA

Petrocelli D., et al, 2017. Procesamiento distribuido y paralelo de bajo costo basado en cloud\&movil. XXIII Congreso Argentino de Ciencias de la Computación, pp 216-225.

Petrocelli D., et al, 2019. Hybrid Elastic ARM\&Cloud HPC Collaborative Platform for Generic Tasks. En Conference on Cloud Computing and Big Data ( $7^{\text {th }}$ Conference, pp.16-27) Communications in Computer and Information Science, Volume 1050. Springer, Cham. Recuperado de https://link.springer.com/chapter/10.1007/978-3-030-27713-0_2

Pramanik P, et al, 2019. Power Consumption Analysis, Measurement, Management, and Issues: A State-of-the-Art Review of Smartphone Battery and Energy Usage. IEEE Access, Volume 7, pp. 182113-182172.

Raake A., et al, 2017. A Bitstream-based, Scalable Video-Quality Model for HTTP Adaptive Streaming: ITU-T P.1203.1. Ninth International Conference on Quality of Multimedia Experience (QoMEX), Erfurt, pp. 1-6.

$\mathrm{Rad}$ B., et al, 2017. An Introduction to Docker and Analysis of its Performance. IJCSNS International Journal of Computer Science and Network Security, Volume 17 - No.3.

Rakesh R., et al, 2020. Bitstream-based Model Standard for 4K/UHD: ITU-T P.1204.3 - Model Details, Evaluation, Analysis and Open Source Implementation. Twelfth International Conference on Quality of Multimedia Experience (QoMEX), Athlone, Ireland, pp 1-6.

Rudrabhatla C., 2020. Security Design Patterns in Distributed Microservice Architecture. International Journal of Computer Science and Information Security (IJCSIS), Volume 18 - No. 7, pp 72-75

Sani Y., et al, 2017. Adaptive Bitrate Selection: A Survey. IEEE Communications Surveys \& Tutorials, Volume 19 No. 4, pp 2985-3014.

Schaffner B., et al, 2018. Smartphones as Alternative Cloud Computing Engines: Benefits and Trade-offs. 2018 IEEE 6th International Conference on Future Internet of Things and Cloud (FiCloud), pp 244-250.

Shi Y. y Sun H., 2019. Image and video compression for multimedia engineering: fundamentals, algorithms, and standards Third Edition (Image Processing Series). CRC Press, Florida, USA.

Shkuro Y., 2019. Mastering Distributed Tracing - Analyzing performance in microservices and complex systems. Packt Publishing Ltd, Birmingham, UK

Shuja J. et al, 2019. Thermal-Efficiency Benchmark on High-Performance Clusters. 2019 Tenth International Green and Sustainable Computing Conference (IGSC), Alexandria, VA, USA, Volume 1, pp 1-8.

Thanh-Tung N. et al., 2020. Horizontal Pod Autoscaling in Kubernetes for Elastic Container Orchestration. Sensors (Basel), Volume 20 - No. 16

Townend P., et al, 2019. Improving Data Center Efficiency Through Holistic Scheduling In Kubernetes. 2019 IEEE International Conference on Service-Oriented System Engineering (SOSE), pp 156-166

Vayghan L., et al, 2019. Microservice Based Architecture: Towards High-Availability for Stateful Applications with Kubernetes. 2019 IEEE 19th International Conference on Software Quality, Reliability and Security (QRS), Sofia, Bulgaria, pp. 176-185.

Watada J., et al, 2020. Emerging Trends, Techniques and Open Issues of Containerization: A Review. IEEE Access, Volume 7, pp. 152443-152472.

Zaman S. et al, 2019. A Systems Overview of Commercial Data Centers: Initial Energy and Cost Analysis. International Journal of Information Technology and Web Engineering, Volume 14 - No. 1, pp 42-65.

Zhong Z. y Buyya R., 2020. A Cost-Efficient Container Orchestration Strategy in Kubernetes-Based Cloud Computing Infrastructures with Heterogeneous Resources. ACM Transactions on Internet Technology, Volume 20 - No 2 , pp 1-24. Article No: 15

Zouheir D. y Hassan H., 2018. Cloud Storage Comparative Analysis Amazon Simple Storage vs. Microsoft Azure Blob Storage. International Journal of Machine Learning and Computing, Volume 8, pages 85-89 